\title{
Criança com anomalia congênita: estudo bibliográfico de publicações na área de enfermagem pediátrica
}

\author{
Child with congenital abnormality: bibliographical study about pediatric nursing publications
}

\author{
Niño con anomalía congénita: estudio bibliográfico sobre publicaciones en el área de enfermería \\ pediátrica
}

\section{Cristiana Araújo Guiller', Giselle Dupas², Myriam Aparecida Mandetta Pettengill ${ }^{3}$}

\section{RESUMO}

Objetivo: conhecer o que tem sido publicado na área de enfermagem sobre a criança com anomalia congênita. Métodos: foi realizada uma revisão bibliográfica em bases de dados indexadas no período de 1995 a 2005. Resultados: os dados foram categorizados em cinco áreas temáticas: exposição da população a fatores de risco para ocorrência de anomalia congênita; uso de tecnologia para o cuidado à criança com anomalia congênita; avaliação de programas de assistência à criança com anomalia congênita e sua família; capacitação do enfermeiro para cuidar da criança com anomalia congênita; e vivências do profissional enfermeiro, da criança e da família com a anomalia congênita. Conclusão: o enfermeiro tem buscado fundamentação teórica e prática para cuidar da criança com anomalia congênita, mas existem lacunas no conhecimento em relação às propostas de intervenções de enfermagem na família da criança com anomalia congênita.

Descritores: Bibliometria Doenças congênitas, hereditárias e neonatais e anormalidades; Enfermagem pediátrica; Publicações científicas e técnicas

\begin{abstract}
Objective: To identity pediatric nursing publications regarding children with congenital defects. Methods: This study was a literature review of articles published in the main health care databases from 1995 to 2005. Results: The retrieved publications were organized into five themes: 1) exposition to risk factors for congenital defects, 2) use of technology to provide care to children with congenital defects, 3) evaluation of the delivery of care to children with congenital defects and their family, 4) nurse's competence to provide quality care to children with congenital defects, and 5) reports of lived experiences of professional nurses who provide care to children with congenital defects, and lived experience of children with congenital defects and their family. Conclusion: Nurses who provide care to children with congenital defects base their practice on strong theoretical and practical foundations; and, evidence based nursing interventions to provide the most efficacious care to children with congenital defects and their family continues to evolve.
\end{abstract}

Keywords: Bibliometrics Congenital, hereditary, and neonatal diseases and abnormalities; Pediatric nursing; Scientific and technical publications

\section{RESUMEN}

Objetivo: conocer qué ha sido publicado en el área de enfermería sobre el niño con anomalía congénita. Métodos: Se realizó una revisión bibliográfica de la literatura, en bases de datos indexadas, en el período de 1995 a 2005 . Resultados: los datos fueron categorizados en cinco áreas temáticas: exposición de la población a factores de riesgo para que ocurra una anomalía congénita; uso de tecnología para el cuidado al niño con anomalía congénita; evaluación de programas de asistencia al niño con anomalía congénita y su familia; capacitación del enfermero para cuidar al niño con anomalía congénita; y vivencias del profesional enfermero, del niño y de la familia con la anomalía congénita. Conclusión: el enfermero ha buscado fundamentos teóricos y prácticos para cuidar al niño con anomalía congénita, sin embargo hay lagunas en el conocimiento en relación a las propuestas de intervenciones de enfermería con la familia del niño con anomalía congénita.

Descriptores: Bibliometría; Enfermedades neonatales congénitas y hereditarias y anomalías; Publicaciones científicas y técnicas; Enfermería pediátrica

\footnotetext{
${ }^{1}$ Mestranda em Enfermagem da Universidade Federal de São Paulo-UNIFESP- (SP), Brasil.

${ }^{2}$ Doutora em Enfermagem. Professora Adjunto do Departamento de Enfermagem da Universidade Federal de São Carlos-UFSCAR-São Carlos (SP), Brasil.

${ }^{3}$ Doutora em Enfermagem. Professora Adjunto do Departamento de Enfermagem da Universidade Federal de São Paulo-UNIFESP- (SP), Brasil.
} 


\section{INDRODUÇÃO}

Em decorrência dos avanços tecnológicos para melhoria nas condições de nascimento e de assistência à saúde infantil, observa-se que está ocorrendo um aumento na sobrevida de crianças que nascem com algum tipo de anomalia congênita.

$\mathrm{Na}$ literatura há diversas definições para anomalia congênita, sendo que para este estudo, adotamos a concepção que a define como "uma condição determinada por fatores causais que atuam antes do nascimento, cuja expressão clínica inclui defeitos em um ou mais órgãos, que podem ser estruturais ou funcionais". Segundo o autor, apesar do termo congênito significar que o defeito básico encontra-se ao nascer, nem sempre é possível demonstrá-lo nos recém-nascidos, já que pode se manifestar, clinicamente, mais tarde ${ }^{(1)}$.

O impacto das anomalias congênitas na saúde do indivíduo, na família e na sociedade é complexo, porque estas patologias são de natureza crônica e podem afetar muitos órgãos e sistemas. Além disso, os problemas médicos, psicológicos e econômicos, entre outros, que esta família deve enfrentar são enormes, sendo imprescindível que todo programa de saúde inclua estratégias de prevenção voltadas para os defeitos congênitos ${ }^{(2)}$.

Em nosso cotidiano, atuando em uma unidade de internação neonatal, observamos o quanto é difícil para os profissionais de saúde o enfrentamento dessa situação, em razão da sua complexidade. Por se tratar de um hospital terciário, de referência para o atendimento de gestações de alto risco, há um grande número de nascimentos de crianças com algum tipo de anomalia congênita, o que desencadeia nos profissionais de saúde, a preocupação em garantir e ampliar a qualidade do cuidado prestado a essas crianças e respectivas famílias.

Com o objetivo de auxiliar o enfermeiro no planejamento e execução de ações de cuidado a essas crianças e famílias, buscamos conhecer o que tem sido publicado sobre essa temática na literatura da área, pois consideramos imprescindível que o enfermeiro fundamente suas ações com evidências geradas por estudos.

Dessa forma, nos motivamos a realizar um estudo bibliográfico de publicações nacionais e internacionais, na área de enfermagem, relacionados à criança com anomalia congênita e sua família, a fim de identificar o conhecimento disponível e verificar possíveis lacunas existentes na área, para subsidiar e direcionar nossa prática clínica com essa clientela.

\section{MÉTODOS}

Para atingir o objetivo, realizamos uma pesquisa exploratória descritiva, por meio de revisão da literatura.

Em um primeiro momento, localizamos publicações que correspondiam aos seguintes critérios de inclusão: ser artigo de pesquisa em periódico nacional ou internacional em inglês ou espanhol; indexados em bases de dados informatizados; ter sido publicado no período de 1995 a 2005; e ter ao menos um profissional enfermeiro como autor.

Consideramos artigo de pesquisa, aquele que divulga os resultados de uma atividade de investigação, organizado conforme metodologia científica ${ }^{(3)}$.

As palavras-chave como indexadores da busca, registradas na Bireme, foram: enfermagem; congênita; anormalidades; malformações e síndrome, sendo que os três últimos foram cruzados um a um com os dois primeiros. Decidimos, por ocasião desse primeiro levantamento, não incluir as palavras-chave criança e família, na tentativa de conseguir maior número de publicações a respeito da temática.

Foram consultadas as bases de dados informatizadas: CINAHL (International Cumulative Index to Nursing and Allied Health Literature); Cochrane Library; PubMed (National Library of Medicine); LILACS (Literatura Latino Americana e do Caribe em Ciências da Saúde); BDENF (Base de Dados de Enfermagem- Bireme); Dedalus e Perienf (contendo acervo de Obras da USP).

Após a busca e seleção das publicações que atendiam aos critérios de inclusão, foram realizadas leitura e releitura das mesmas, a fim de extrair os pontos centrais. Em seguida foi realizada uma segunda leitura, buscando-se extrair as similaridades e divergências dos estudos, sendo realizado um fichamento de cada artigo, a fim de organizar os dados. Nesse momento, os dados extraídos foram agrupados em categorias ou áreas temáticas, a fim de alcançar uma análise a respeito dos estudos consultados.

\section{RESULTADOS}

Foram encontradas 48 publicações nacionais e 106 internacionais sobre a temática. Destas, foram selecionados 41 artigos de pesquisa, sendo 24 artigos de periódicos nacionais e 17 internacionais que correspondiam aos critérios de inclusão previamente estabelecidos.

Os estudos foram categorizados em unidades temáticas, assim denominadas: exposição da população a fatores de risco para ocorrência de anomalia congênita; uso de tecnologia para o cuidado à criança com anomalia congênita; avaliação de programas de assistência à criança com anomalia congênita e sua família; capacitação do enfermeiro para cuidar da criança com anomalia congênita; e vivências do profissional enfermeiro, da criança e da família com a anomalia congênita.

\section{DISCUSSÃO}

Apresentamos, a seguir, os agrupamentos temáticos 
que emergiram da análise qualitativa realizada sobre as publicações:

\section{Exposição da população a fatores de risco para ocorrência de anomalia congênita}

Dez estudos foram categorizados nessa temática cujo objetivo foi identificar como a população se expõe aos possíveis fatores de risco para ocorrência de anomalias congênitas, além de avaliar a qualidade da assistência oferecida no pré-natal pelos serviços de saúde.

Fatores de risco para ocorrência de anomalia congênita estudados são doenças como rubéola e sífilis congênita, que ainda são uma ameaça a gestantes, e trazem conseqüências graves como abortos e anomalias congênitas a seus conceptos ${ }^{(4-7)}$. Quando se avalia o atendimento prestado pelos serviços de saúde neste contexto, percebe-se que a assistência prestada no prénatal, em parte das Unidades Básicas de Saúde (UBS), ainda não atende plenamente às exigências do Ministério de Saúde ${ }^{(5-7)}$.

Outros fatores estudados incluem o uso indevido de fármaco/drogas, revelando que há associação significante entre sua utilização e a presença de anomalias congênitas $^{(8-11)}$.

Pesquisas realizadas em serviços especializados para anomalias congênitas, identificaram que as gestantes, geralmente, procedem da zona rural e possuem fatores de risco como por exemplo uso de medicação para abortar, uso de drogas, idade avançada, filho anterior com anomalia congênita, dentre outros ${ }^{(12-13)}$.

Esses estudos são importantes, pois auxiliam o enfermeiro na prevenção da anomalia congênita por meio de educação em saúde, conscientizando as gestantes em relação aos cuidados que devem ter para evitar possíveis exposições a esses fatores, assim como a vacinação e a prevenção de doenças sexualmente transmissíveis.

\section{Uso de tecnologia para o cuidado à criança com anomalia congênita}

Neste grupo foram categorizados três estudos que enfocam a importância da utilização de recursos tecnológicos para assistir e melhorar a qualidade de vida de crianças com anomalia congênita, bem como para fundamentar a assistência de enfermagem a essa população especificamente.

A anquiloglossia (frênulo lingual pequeno que limita os movimentos da língua) é uma anomalia congênita relativamente freqüente na população de recém-nascidos e representa uma proporção significativa dentre os problemas encontrados para a amamentação. O procedimento de frenuloplastia, em crianças com anquiloglossia, é avaliado em um estudo ${ }^{(14)}$ e identificado como uma técnica que facilita a amamentação, contribuindo para o crescimento e desenvolvimento saudável destas crianças.

Em outro estudo ${ }^{(15)}$, os pesquisadores compararam a saturação de $\mathrm{O}_{2}$, por meio de oxímetro de pulso, em crianças com doença cardíaca congênita amamentadas com leite materno no seio, com outras, nutridas por mamadeira. Como resultados, verificaram que a amamentação no seio materno não reduz a saturação de $\mathrm{O}_{2}$, enquanto que, durante a nutrição por mamadeira, a saturação teve quedas com maior freqüência. Esse resultado é importante, pois indica que a amamentação no seio materno não deve ser contra-indicada para crianças com algum tipo de cardiopatia, como vinha sendo preconizado por outros autores na literatura.

$\mathrm{O}$ uso da tecnologia, em algumas situações pode acarretar problemas, como o revelado no estudo que buscou avaliar o grau de ansiedade materna, após a realização do teste para diagnóstico intra-útero de Síndrome de Down. Como resultado, os autores perceberam que as mulheres que se submetem ao teste ficam mais ansiosas e preocupadas com seu bebê. Recomendam que, ao se introduzir este tipo de exame fetal, é necessário que sejam incluídas intervenções adequadas, a fim de não aumentar a carga de ansiedade materna, que pode persistir no período restante da gestação $^{(16)}$.

Avaliação de programas de assistência à criança com anomalia congênita e sua família

Neste agrupamento, os três estudos categorizados têm como foco a avaliação dos programas direcionados à assistência da criança com anomalia congênita e sua família. O enfermeiro busca conhecer os resultados das atividades que estão desenvolvendo junto à clientela, como forma de planejarem suas ações e ampliarem as ofertas de atendimento.

Em dois estudos realizados para avaliar programas que visam oferecer suporte às famílias durante o processo de adaptação aos filhos, os autores observaram que pais participantes apresentam menor nível de estresse, de ansiedade e de depressão, além de percepções e atitudes mais positivas em relação à doença da criança e sua situação. Esses pais também se revelam satisfeitos por terem a oportunidade de expressar seus sentimentos e dificuldades, sentindo-se menos sozinhos ${ }^{(17-18)}$.

Um artigo mexicano avaliou o Programa de Triagem Neonatal realçando a importância da detecção precoce de doenças congênitas como prática cotidiana e a necessidade de ampliá-lo para que seja garantido a todos os recém-nascidos. Os autores observaram um aumento significativo do número de cobertura de exames para a população de neonatos ${ }^{(19)}$.

Esses estudos norteiam o que tem sido realizado e ajudam na elaboração de novas propostas de atendimento a essa população. 


\section{Capacitação do enfermeiro para cuidar da criança com anomalia congênita}

Neste grupo, os quatro artigos categorizados tiveram por objetivo a proposição de roteiros sistematizados para assistir crianças com anomalia congênita, com base em revisão da literatura e experiência clinica na prática hospitalar.

O foco dos estudos foi ampliar a capacitação do enfermeiro, fornecendo informações acerca dos cuidados específicos para crianças com anomalia congênita, em especial, a atresia de esôfago, cardiopatia congênita e fenda de lábio e/ou palato ${ }^{(20-23)}$.

Tais estudos revelam a importância do papel que o profissional de enfermagem exerce ao cuidar destas crianças, daí a necessidade de ampliar seu conhecimento científico.

\section{Vivências do enfermeiro, da criança e da família com a anomalia congênita}

Neste agrupamento foram categorizadas 21 publicações que se referiam à vivência dos sujeitos envolvidos com o cuidado e tratamento da criança com anomalia congênita, assim como da própria criança, com o objetivo de compreender o significado dessa experiência e delinear estratégias de cuidado que contemplem suas necessidades.

Os estudos que buscam compreender a experiência vivida pela criança com anomalia congênita, revelam situações complexas em que esta se vê envolvida, tais como: mudanças de ordem prática e cotidiana, angústia mental, abandono, relações afetivas prejudicadas, interações diferenciadas e preconceituosas no contexto em que estão inseridas, dentre outras ${ }^{(24-26)}$.

Pesquisas relacionadas à experiência do enfermeiro na assistência de crianças nascidas com anomalia congênita revelam que o cuidar da criança com anomalia congênita é uma tarefa desgastante do ponto de vista emocional, mas capaz de gerar grande satisfação pessoal, e que, quando há trabalho em equipe, aliado ao conhecimento teórico, as dificuldades podem ser minimizadas e superadas com maior facilidade ${ }^{(27-29)}$.

Estudos sobre a vivência da família da criança com anomalia congênita, têm como ponto em comum o impacto emocional provocado pela comunicação do diagnóstico e do nascimento do bebê. Os autores evidenciaram que nesse momento, há o desencadeamento de sentimentos de culpa e tristeza, por não terem gerado uma criança saudável e que corresponda à cultura de beleza e perfeição estética aceitáveis na sociedade ${ }^{(30-33)}$.

Considerando que a comunicação do diagnóstico é de fundamental importância, pois é o início de um processo de ajustamento para toda a vida da família, diversos autores têm buscado compreender qual é o momento ideal para que o diagnóstico seja revelado à família. Pesquisas com mães, que só souberam do diagnóstico de anomalia congênita após o nascimento, revelam que preferiam ter recebido a notícia o mais cedo possível. Queixam-se da demora da informação acerca do diagnóstico e da forma inadequada de comunicação estabelecida pelos profissionais ${ }^{(34-35)}$. Porém, outro estudo, explorou a situação de pais que recebem o diagnóstico de anomalia congênita durante a gestação, revelando que vivenciam processos de angústia, como a perda do bebê idealizado, e ainda, a difícil situação de ter que decidir por interromper a gestação, quando há incompatibilidade para a vida ${ }^{(36)}$.

Outros estudos mostram que, ainda sob o choque ocasionado pelo diagnóstico, e na tentativa de melhor organizar seu cotidiano diante deste fato inesperado, as famílias seguem em busca de informações a respeito da doença de sua criança e de apoio, formando um amplo vínculo com os profissionais de saúde e com outras pessoas que experienciam a mesma situação ${ }^{(37-39)}$.

Outro aspecto da vivência que tem despertado o interesse de estudiosos é a compreensão do significado de ter uma criança com anomalia congênita. Para os pais, significa se deparar com situações desagradáveis, como não estar habilitado para lidar com a discriminação social; porém vivenciam situações favoráveis quando imaginam que existem condições piores que as suas. Eles se conformam com a situação quando acreditam que ter um filho com deficiência é a vontade de Deus ${ }^{(40)}$.

Há ainda uma preocupação dos estudiosos em identificar diferenças e similaridades entre pais e mães de uma criança com deficiência. Os estudos realizados mostram que há mais diferenças do que similaridades. As mães apresentam-se mais preocupadas com sua relação conjugal e problemas do dia-a-dia em lidar com a criança, buscando atender às necessidades físicas da criança. Os pais se revelam preocupados com questões da família extensa e com o futuro da criança, buscando atender às necessidades psicossociais ${ }^{(41-42)}$.

Outro aspecto estudado é relacionado às principais dificuldades que as famílias enfrentam ao prestarem cuidados às crianças com anomalia congênita em casa. Evidenciam que as orientações recebidas pela família antes da alta não contemplam suas necessidades, favorecendo o surgimento de dúvidas e/ou dificuldades em relação ao cuidado dispensado à criança. Dessa maneira, recomendam que as orientações sejam realizadas antes e após a alta, para que se transmita segurança e minimize a ansiedade destes pais ${ }^{(43-44)}$.

\section{CONCLUSÃO}

Com a realização deste estudo, percebemos que o enfermeiro tem buscado fundamentação teórica e 
prática para cuidar da criança com anomalia congênita e sua família, realizando estudos sobre propostas de intervenção para os principais tipos de anomalias congênitas, bem como de compreensão da experiência de enfermeiros, da própria criança e da família com a anomalia congênita.

A análise temática realizada nos permitiu observar que grande parte das pesquisas de literatura nacional apresenta, como foco, o levantamento de fatores de risco para a ocorrência de anomalias congênitas, e propostas de intervenção centradas em prevenção e tratamento, assim como de protocolos assistenciais para o cuidado.

Tanto na literatura nacional como internacional, foram encontradas pesquisas do tipo qualitativa, que abordam a compreensão do significado da experiência da família, de enfermeiros e da própria criança com a anomalia congênita, mas que estão ainda centrada no impacto do diagnóstico para a família, com enfoque especial na mãe, revelando que, apesar do enfermeiro preocupar-se em incluir a família em sua prática assistencial, há lacunas no conhecimento, principalmente em relação às propostas de intervenções de enfermagem para a família da criança com anomalia congênita, que precisam ser mais bem esclarecidas.

Há questionamentos que permanecem pouco explorados: como a família se reestrutura após o impacto do nascimento de uma criança com anomalia congênita? Como os demais membros da família convivem com essa criança? Como a família organiza seu cotidiano para o cuidado dessa criança? Como o enfermeiro pode ajudar a família no enfrentamento dessa situação?

Acreditamos ser de grande importância a realização de pesquisas de enfermagem com famílias que vivenciam tal situação, a fim de melhor compreendermos sua experiência no processo de cuidar da criança em seu cotidiano. Porém, é preciso investir mais em pesquisas relacionadas a intervenções, a fim de instrumentalizar o enfermeiro para o cuidado e ampliar o corpo de conhecimento teórico da área de enfermagem da família fundamentado em evidências científicas.

\section{REFERÊNCIAS}

1. Reis LV. Anomalias congênitas, identificadas ao nascimento, em filhos de mulheres adolescentes [tese]. São Paulo: Escola Paulista de Medicina. Universidade Federal de São Paulo; 2005.

2. Abrahão AR. Caracterização de uma amostra de gestantes de risco aumentado para anomalias congênitas: aspectos sócio-econômicos, clínicos e tipo de risco [tese]. São Paulo: Escola Paulista de Medicina. Universidade Federal de São Paulo; 1995.

3. Oliveira AL. Produção científica brasileira da área de enfermagem obstétrica de 1956 a 1986 [tese]. São Paulo: Escola Paulista de Medicina. Universidade Federal de São
Paulo; 1992.

4. Barros SMO, Lacava RMVB, Lima MBO. Susceptibilidade à rubéola entre gestantes: prevalência e intervenções de enfermagem. Acta Paul Enfermagem. 2001; 14(1):54-61.

5. Ozaki LMTR, Feracin JCF, Simo AKK. Campanha de vacinação contra a rubéola: mães e filhos correm riscos? Rev Enfermagem UERJ. 2004; 12(1):60-5.

6. Figueiredo EN, Pichineli V, Rossetto MACC, Vianna LAC. Estudo de caso de sífilis congênita. Acta Paul Enfermagem. 2001; 14(3):28-34.

7. Silva LR, Santos RS. O que as mães sabem e sentem sobre a sífilis congênita: um estudo exploratório e suas implicações para a prática de enfermagem. Esc Anna Nery Rev Enferm. 2004; 8(3):393-401.

8. Kaup ZOL, Merighi MAB, Tsunechiro MA. Avaliação do consumo de bebida alcoólica durante a gravidez. Rev Bras Ginecol Obstet. 2001; 23(9):575-80.

9. Souza GT, Rodrigues MC, Ciavaglia MC. Análise do grau de conhecimento da população sobre a teratogenia do álcool e a conduta de enfermagem. Rev Bras Enfermagem. 1996; 49(2):287-304.

10. Barros SMO. Estudo sobre o uso de fármacos durante a gestação. Acta Paul Enfermagem. 1995; 8(1/3):26-32.

11. Barros SMO, Juliano Y, Moron AF. Crescimento e desenvolvimento de fetos humanos expostos ao misoprostol e sua avaliação perinatal. Acta Paul Enfermagem 1998; 11(2):7-19.

12. Abrahão AR, Gomes AA, Santos M. Gestantes de risco para anomalias fetais atendidas no Caenf/setor de medicina fetal- UNIFESP em 1998. Acta Paul Enfermagem. 2000; 13(N Esp Pt 2):205-8.

13. Donato M, Córdoba E. Prevalencia de las malformaciones congénitas en la zona del ramal jujeño. Temas Enferm Actual. 1998; 6(29):5-8.

14. Ballard JL, Auer CE, Khoury JC. Ankyloglossia: assessment, incidence, and effect of frenuloplasty on the breastfeeding dyad. Pediatrics. 2002; 110(5):e63.

15. Marino BL, O`Brien P, LoRe H. Oxygen saturations during breast and bottle feedings in infants with congenital heart disease. J Pediatr Nurs. 1995; 10(6):360-4.

16. Georgsson Ohman S, Saltvedt S, Grunewald C, Waldenstrom U. Does fetal screening affect women's worries about the health of their baby? A randomized controlled trial of ultrasound screening for Down's syndrome versus routine ultrasound screening. Acta Obstet Gynecol Scand. 2004; 83(7):634-40.

17. Pelchat D, Bisson J, Ricard N, Perreault M, Bouchard JM. Longitudinal effects of an early family intervention programme on the adaptation of parents of children with a disability. Int J Nurs Stud. 1999; 36(6):465-77.

18. Pelchat D, Lefebvre H, Proulx M, Reidy M. Parental satisfaction with an early family intervention program. J Perinat Neonatal Nurs. 2004; 18(2):128-44.

19. Dámaso Ortíz B. Participación del personal de enfermería en la toma de tamiz neonatal para la detección de hipotiroidismo congénito. Bol Méd Hosp Infant Mex. 2001; 58(11):755-61.

20. Gentil RC, Reis MCF, Saiki J, Samezima CMH. Perfil de crianças com cardiopatia congênita que utilizaram o Serviço de Remoção Aeromédica. Acta Paul Enfermagem. 2003; 16(3):51-61.

21. Falcão MC, Pacios RAS. Abordagem multidisciplinar do 
recém-nascido com atresia de esôfago. Rev Paul Pediatr. 1997; 15(4):181-6.

22. Jansen D, Silva KVPT, Novello R, Guimarães TCF, Silva VG. Assistência de enfermagem à criança portadora de cardiopatia. Rev SOCERJ. 2000; 13(1):22-9.

23. Araruna RC, Vendrúscolo DMS. Alimentação da criança com fissura de lábio e/ou palato- um estudo bibliográfico. Rev Latinoam Enfermagem. 2000; 8(2):99-105.

24. Mobley CE, Harless LS, Miller KL. Self-perceptions of preschool children with spina bifida. J Pediatr Nurs. 1996; 11(4):217- 24.

25. Furlan MFFM, Ferriani MGC, Gomes R. O cuidar de crianças portadoras debexiga neurogênica: representações sociais das necessidades dessas crianças e suas mães. Rev Latinoam Enfermagem. 2003; 11(6):763-70.

26. Barreire SG, Oliveira OA, Kazama W, Kimura M, Santos VLCG. Qualidade de vida de crianças ostomizadas na ótica das crianças e das mães. J Pediatr (Rio de Janeiro). 2003; 79(1):55-62.

27. Spiri WC, Leite MMJ. O significado do trabalho em equipe na reabilitação de pessoas com malformação craniofacial congênita. Rev Esc Enfermagem USP. 2004; 38(3):288-97.

28. Negri MDX, Labronici LM, Zagonel IPS. O cuidado inclusivo de enfermagem ao portador da Síndrome de Down sob o olhar de Paterson e Zderad. Rev Bras Enfermagem. 2003; 56(6):678-82.

29. Farrell R, Bennett L, Dixon RM. The impact of the physical appearance of dying babies on the attitudes and reactions of nurses in neonatal intensive care units. Neonat Paediatr Child Health Nurs. 2000; 3(4):4-11.

30. Pinheiro MCD. O "ser-mãe" de criança com malformação: um estudo fenomenológico. Rev Bras Enfermagem. 1997; 50(2):197-214.

31. Horta ALM. Significado do nascimento de uma criança com cardiopatia para a família. Acta Paul Enfermagem. 1996; 9(N Esp):119.

32. Rillstone P, Hutchinson SA. Managing the reemergence of anguish: pregnancy after a loss due to anomalies. J Obstet Gynecol Neonatal Nurs. 2001; 30(3):291- 8.

33. Raines DA. Suspended mothering: women's experiences mothering an infant with a genetic anomaly identified at birth. Neonatal Netw. 1999; 18(5):35-9.

34. Kersting LR, Prevedello ML, Socal ES, Silva AS. Conhecimentos e sentimentos de mães de crianças portadoras da síndrome de down e a atuação da enfermagem. Disci-plinarum Scientia. 2000; (1):1-11.

35. Rubio Rubio SA, Santos RS. Mães de crianças com anomalia congênita: histórias de gestantes peruanas assistidas em situações especiais. Esc Anna Nery Rev Enferm. 1999; 3(2):69-80.

36. Bryar SH. One day you're pregnant and one day you're not: pregnancy interruption for fetal anomalies. J Obstet Gynecol Neonatal Nurs. 1997; 26(5):559-66.

37. Sigaud CHS, Reis AOA. A representação social da mãe acerca da criança com Síndrome de Down. Rev Esc Enfermagem USP. 1999; 33(2):148-56.

38. Higarashi IH, Pelloso SM, Carvalho MDB. Ser mãe de uma criança com Síndrome de Down: uma visão compreensiva. Nursing (São Paulo). 1998; 1(6):21-5.

39. Monsen RB. Mothers' experiences of living worried when parenting children with spina bifida. J Pediatr Nurs. 1999; 14(3):157-63.

40. Baumann SL, Braddick M. Out of their element: fathers of children who are "not the same". J Pediatr Nurs. 1999; 14(6):369-78.

41. Svavarsdottir EK, McCubbin M. Parenthood transition for parents of an infant diagnosed with a congenital heart condition. J Pediatr Nurs. 1996; 11(4):207-16.

42. Pelchat D, Lefebvre H, Perreault M. Differences and similarities between mothers' and fathers' experiences of parenting a child with a disability. J Child Health Care. 2003; 7(4):231-47.

43. Maresca M, Giovani AMM. Levantamento de dados para elaboração do manual de orientações para cuidadores de crianças em período de tratamento conservador da displasia do desenvolvimento do quadril. Rev Enfermagem do Complexo HCFMUSP. 1999; 3(4):14-8.

44. Hilbert GA, Walker MB, Rinehart J. "In for the long haul": responses of parents caring for children with Sturge-Weber Syndrome. J Fam Nurs. 2000; 6(2):157-79. 